\title{
Lattice Vibrations and Superconductivity in Layered Structures*
}

\author{
R. E. Allen, G. P. Alldredge, and F. W. de Wette \\ Department of Physics, University of Texas, Austin, Texas 78712 \\ (Received 18 May 1970)
}

\begin{abstract}
In order to estimate the influence of both surface and interface effects on phonon frequencies and superconducting transition temperatures in layered structures, we have calculated the vibrational modes of structures composed of alternating films of heavy and light particles. An interface effectively lowers the vibrational frequencies of the heavy particles and raises those of the light particles. Interface effects are small compared to surface effects, however. The decrease in the frequencies for a three-layer film on a substrate (with respect to the bulk frequencies) is only $40-50 \%$ of the decrease for a free three-layer film.
\end{abstract}

About two years ago, Strongin et al. ${ }^{1}$ found large increases in superconducting transition temperatures $\left(T_{c}\right)$ for layered structures consisting of alternating films of dissimilar metals. The hypothesis used to explain this result was a decrease in the phonon frequencies due to surface and interface effects. This hypothesis has several sources of support. First, it is in accordance with the qualitative behavior of the experimental results. (There is an initial rise in $T_{c}$ for a thickness of less than about three monolayers, which can be explained by the proximity effect, ${ }^{2}$ and than a decrease in $T_{c}$ which can be attributed to a decrease in the relative importance of surface and interface effects. ) Second, the results of McMillan ${ }^{3}$ suggest that changes in the phonon frequencies are of primary importance in determining changes in $T_{c}$, at least for some materials. Third, there is good evidence that changes in $T_{c}$ for disordered and amorphous materials are due mainly to changes in the phonon frequencies. ${ }^{4,5}$ Finally, Dickey and Paskin $^{6}$ and the present authors ${ }^{7}$ have calculated the vibrational frequencies in free crystalline films; these calculations gave results which, when substituted into expressions for $T_{c}$ developed by McMillan ${ }^{3}$ and Garland et al., ${ }^{4}$ yield increases in $T_{c}$ of the same order of magnitude as the observed increases.

One unrealistic feature of these calculations, however, is that they are for free films, whereas the experimental films lie on a substrate. (Another possible deficiency is the assumption of a crystalline structure and planar surfaces, whereas the actual structure of the films is unknown at the time this is written. ${ }^{2}$ Before the proper structure can be taken into account, however, it must be determined experimentally. )

In order to estimate the influence of both interface and surface effects on $T_{c}$, we have calculated the vibrational modes of layered structures in which the films are in contact with one another.
The structures for which the present results were obtained consist of seven films, each composed of three monolayers of identical particles, stacked atop one another to form a 21-layer slab. A side view of this system is shown in Fig. 1. The films alternately have atomic masses $m$ and $M$, with $m$ corresponding to the outermost films. The crystal structure (fcc) and interaction between particles (Lennard-Jones 12-6 with the same potential parameters) are assumed to be identical for both types of particle; only the masses are different.

The frequencies $\omega$ for such a system are calculated by solving the matrix eigenvalue equation ${ }^{8}$

$$
\sum_{l_{3}^{\prime} \beta} D_{\alpha \beta}\left(l_{3} l_{3}^{\prime}\right) \xi_{\beta}\left(l_{3}^{\prime} ; \omega\right)=\omega^{2} \xi_{\alpha}\left(l_{3} ; \omega\right),
$$

where

$$
\begin{aligned}
D_{\alpha \beta}\left(l_{3} l_{3}^{\prime}\right)= & {\left[M\left(l_{3}\right) M\left(l_{3}^{\prime}\right)\right]^{-1 / 2} } \\
& \times \sum_{l^{\prime}} \Phi_{\alpha \beta}\left(\overrightarrow{1}^{\prime}\right) \exp \left[i q \cdot\left(r_{0}^{l}-r_{0}^{l^{\prime}}\right)\right] .
\end{aligned}
$$

$M\left(l_{3}\right)$ is the mass of a particle in the monolayer labeled by $l_{3}^{\prime}, \xi_{\alpha}\left(l_{3} ; \omega\right)$ gives the amplitude of vibra-

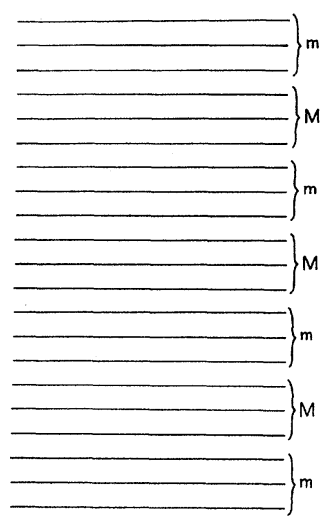

FIG. 1. Side view of layered structure consisting of three-layer films with masses $m$ and $M$. 
tion in the $\alpha$ direction $(\alpha=x, y$, or $z$ ) for a particle in this monolayer vibrating in the mode with frequency $\omega$, and the other symbols have been defined previously. ${ }^{9}$ As before, ${ }^{7}$ all the quantities with which we are presently concerned (e.g., $\omega$ ) can be scaled in terms of $M$ and the potential parameters $\sigma$ and $\epsilon$, so we will take $M=\sigma=\epsilon=1$ for simplicity in presenting these results. Finally, we mention that the results given here are for the density of a static crystal $(\sigma / a=1.29724$, where $\sqrt{2} a=$ nearest-neighbor distance) and for a (111) surface.

In the approximate expressions that have been developed for the transition temperature of an iso lated system, ${ }^{3,4}$ the following average over the phonon frequency distribution is important:

$$
\begin{aligned}
\left\langle\omega^{2}\right\rangle_{M} & \equiv \int_{0}^{\omega} \alpha^{2}(\omega) f(\omega) \omega d \omega / \int_{0}^{\omega} \alpha^{2}(\omega) f(\omega) \omega^{-1} d \omega \\
& \approx\langle\omega\rangle /\left\langle\omega^{-1}\right\rangle
\end{aligned}
$$

where

$$
\left\langle\omega^{n}\right\rangle \equiv \int_{0}^{\omega_{0}} f(\omega) \omega^{n} d \omega=(1 / N) \sum_{\omega} \omega^{n}
$$

with $N$ representing the number of vibrational frequencies $\omega$ occuring in the summation (and the limit $N \rightarrow \infty$ assumed). Here $\omega_{0}$ is the maximum frequency, $f(\omega) d \omega$ is proportional to the number of frequencies lying between $\omega$ and $(\omega+d \omega)$ [with $f(\omega)$ normalized to unity], and $\alpha^{2}(\omega)$ is an average of the electron-phonon matrix element squared. The quantity $\left\langle\omega^{2}\right\rangle_{M}$ determines the electron-phonon coupling constant $\lambda$ through the equation ${ }^{3}$

$$
\lambda=N(0)\left\langle\boldsymbol{g}^{2}\right\rangle / \mathfrak{T}\left\langle\omega^{2}\right\rangle_{M^{\prime}}
$$

and, in the approximation of Garland and Allen, ${ }^{4}$ it determines $T_{c}$ by the equation

$$
T_{c}=\frac{\hbar \omega_{0}}{k_{B}}(0.25+0.67 x) \exp \left(-\frac{1+\lambda}{\left.\left(0.53+0.62 x^{1 / 2}\right) \lambda-\mu^{*}-(0.25+0.67 x) \lambda \mu^{*}\right)}\right)
$$

where $x \equiv\left\langle\omega^{2}\right\rangle_{M} / \omega_{0}^{2}$. In Eq. (5), $\mathfrak{N}$ is the atomic mass and the other symbols have been previously defined. $^{3,7}$

In the case of a layered structure, however, we are not interested in the value of this moment over the whole system. Presumably, the electrons responsible for the enhanced transition temperature are localized primarily in the outermost film, and their phonon-mediated interaction should primarily involve the vibrations of particles in this film. We therefore define an effective moment for a single film,

$$
\left\langle\omega^{n}\right\rangle_{f}=\frac{N_{3}}{N_{f}} \frac{1}{N} \sum_{\omega} \omega^{n}\left(\sum_{l_{3}(\text { in } 111 \mathrm{~m})} \sum_{\alpha}\left|\xi_{\alpha}\left(l_{3} ; \omega\right)\right|^{2}\right) .
$$

That is, in determining this moment, each frequency $\omega$ is weighted according to how much amplitude its mode has within the film; specifically, the weight is equal to the sum of $\left|\xi_{\alpha}\left(l_{3} ; \omega\right)\right|^{2}$ within the film. In Eq. (7), $N_{3}$ is the number of monolayers in the whole system and $N_{f}$ is the number in the film. (In the present case, $N_{3}=21$ and $N_{f}=3$.) We introduce the factor $N_{3} / N_{f}$ in order to give a normalization equivalent to that used in Eq. (4). For example, $\left\langle\omega^{0}\right\rangle=1$; with the definition of Eq. (7) and the closure condition on the $\xi_{\alpha}\left(l_{3} ; \omega\right)$, it turns out that $\left\langle\omega^{0}\right\rangle_{f}=1$ also.

It is also useful to define an effective frequency distribution function $f_{\alpha}\left(\omega ; l_{3}\right)$ such that

$$
f_{\alpha}\left(\omega ; l_{3}\right) d \omega=\frac{3 N_{3}}{N} \sum_{\omega \leqslant \omega^{\prime}<\omega+d \omega}\left|\xi_{\alpha}\left(l_{3} ; \omega^{\prime}\right)\right|^{2} .
$$

That is, the contribution of a frequency to $f_{\alpha}\left(\omega ; l_{3}\right)$ is weighted according to how much the corresponding vibrational mode is represented in the $l_{3}$ th layer and the $\alpha$ direction. [The factor of $3 N_{3}$ is inserted in order to normalize $f_{\alpha}\left(\omega ; l_{3}\right)$ to unity.]

In Fig. 2, results are shown for both the frequency distribution function $f(\omega)$ and some of the effective distribution functions $f_{\alpha}\left(\omega ; l_{3}\right)$ in the case of a layered structure with masses 1 and $\frac{1}{5}$ (i.e., $m=\frac{1}{5}$ in Fig. 1). Figures $2(\mathrm{a})$ and 2(b), respectively, show $f(\omega)$ for a monatomic slab $(m=1)$ and for the layered structure. It can be seen that $f(\omega)$ for the layered structure consists roughly of a superposition of $f(\omega)$ 's for monatomic crystals with masses of 1 and $\frac{1}{5}$.

In Figs. 2(c) and 2(d), the effective distribution functions $f_{\alpha}\left(l_{3} ; \omega\right)$ are shown for $\alpha=x$ and $z$, respectively, and for $l_{3}$ corresponding to the outermost monolayer. ${ }^{10}$ (The $x$ and $z$ directions are, respectively, parallel and perpendicular to the surface. ) It can be seen that the effective $f(\omega)$ for in-plane vibrations is very similar to the $f(\omega)$ for a monatomic crystal. The effective $f(\omega)$ for vibrations perpendicular to the surface, however, contains a large peak at low frequencies which is due to the presence of Rayleigh-surface modes. This peak is almost identical to the one which is obtained for a monatomic crystal, i. e., the vibrations in the surface monolayer are almost the same for the layered structure and a monatomic crystal.

Figures 2(e) and 2(f) contain results for the third 
monolayer beneath the surface (last layer in the outermost film). Again, in-plane vibrations are not much different from those in an infinite monatomic crystal. However, the vibrations perpendicular to the interface are quite different, and it can be seen that the effect of the interface is to raise the vibrational frequencies (in the sense that the average frequency is raised).

In Figs. 2(g) and 2(h), the effective $f(\omega)$ 's are shown for the fourth monolayer beneath the surface (first layer in the next-to-outermost film). The in-plane vibrations are not much affected by the interface. The perpendicular vibrations are again considerably affected, and the effect is to lower the frequencies. They are not lowered as much as at a surface, however; whereas the surface mode peak for a mass of 1 occurs at $\omega \approx 11,{ }^{11}$ the "interface mode" peak occurs at $\omega \approx 17$.

The fact that the frequencies are lowered for the heavier particles at an interface can be attributed to the fact that the lighter particles provide relatively little resistance to the motion of the heavier ones. ${ }^{12}$ So far as the lighter particles are concerned, however, the interface provides a nearly rigid boundary (for a mass ratio much different from unity), and so their frequencies are increased above those of an infinite monatomic crystal.

We do not show the effective $f(\omega)$ 's for the other layers in the system. It is found, however, that the results at all the interfaces are similar to those shown in Figs. 2(e)-2(h), i. e., vibrations at the first interface are not much affected by the presence of the surface, just as vibrations at the surface are not much affected by the presence of the interfaces. The middle layer in each film shows relatively weak interface (or surface) effects.

In Table I, some effective moments $\left\langle\omega^{n}\right\rangle_{f}$ for a three-layer film are given for a number of different situations, i.e., for various mass ratios and for films either at the surface (so that there are both surface and interface effects) or well beneath the surface (so that there are only interface effects).
TABLE I. Effective moments for three-layer films under eight conditions: (a) Film is at surface of layered structure, mass ratio $=1 / 5$. (In all cases, the mass ratio is the ratio of the atomic mass for the film under consideration to that for the films with which it has interfaces.) (b) Film at surface, mass ratio $=1 / 2$. (c) Film at surface, mass ratio $=2 / 1$. (d) Film has two free surfaces. (e) Film is in interior of structure, well beneath the surface, and mass ratio $=1 / 5$. (f) Film in interior, mass ratio $=1 / 2$. (g) Film in interior, mass ratio $=2 / 1$. (h) Film in interior, mass ratio $=5 / 1$. The values given are the ratios $\left\langle\omega^{n}\right\rangle_{f} /\left\langle\omega^{n}\right\rangle_{\text {bulk }}$, where $\left\langle\omega^{n}\right\rangle_{f}$ is defined by Eq. (7) and $\left\langle\omega^{n}\right\rangle_{\text {bulk }}$ is the moment calculated for the bulk (Ref. 7) using Eq. (4).

\begin{tabular}{|c|c|c|c|c|}
\hline & & $\frac{\langle\omega\rangle_{f}}{\langle\omega\rangle_{\text {bu1k }}}$ & $\frac{\left\langle\omega^{2}\right\rangle_{f}}{\left\langle\omega^{2}\right\rangle_{\mathrm{bu} 1 \mathrm{k}}}$ & $\frac{\langle\omega\rangle_{f}}{\langle\omega\rangle_{\text {bulk }}} \frac{\left\langle\left\langle\omega^{-1}\right\rangle_{f}\right.}{/\left\langle\omega^{-1}\right\rangle_{\text {bulk }}}$ \\
\hline (a) & $\begin{array}{l}\text { surface, } \\
1 / 5\end{array}$ & 0.937 & 0.891 & 0.852 \\
\hline (b) & $\begin{array}{l}\text { surface, } \\
1 / 2\end{array}$ & 0.936 & 0.891 & 0.847 \\
\hline (c) & $\begin{array}{l}\text { surface, } \\
2 / 1\end{array}$ & 0.933 & 0.891 & 0.840 \\
\hline (d) & free film & 0.870 & 0.789 & 0.650 \\
\hline (e) & $\begin{array}{l}\text { interior, } \\
1 / 5\end{array}$ & 1.005 & 1.000 & 1.021 \\
\hline (f) & $\begin{array}{l}\text { interior, } \\
1 / 2\end{array}$ & 1.002 & 1.000 & 1.010 \\
\hline (g) & $\begin{array}{l}\text { interior, } \\
2 / 1\end{array}$ & 0.997 & 1.000 & 0.995 \\
\hline (h) & $\begin{array}{l}\text { interior, } \\
5 / 1\end{array}$ & 0.992 & 1.000 & 0.986 \\
\hline
\end{tabular}

For comparison, the results for a free threelayer film are also shown in Table I. It is evident that the decrease in the vibrational frequencies, relative to the bulk frequencies, is far less for a film on a substrate than for a free film. In fact, the ratio of the decreases in $\langle\omega\rangle_{f} /\left\langle\omega^{-1}\right\rangle_{f}$ is about $45 \%$ (for a mass ratio of about 1 ), in accordance with the expectation that replacing two free surfaces by a surface and an interface will reduce the size of surface effects by about $50 \%$. The further reduction of about $5 \%$ can be attributed to effects of the small thickness of a three-layer film: The relaxation of surface particles is larger than for

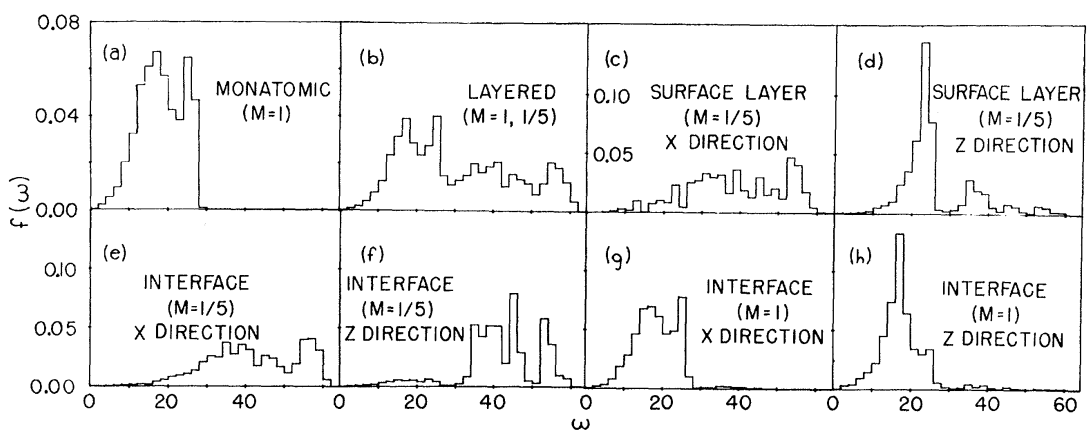

FIG. 2. Frequency distribution function $f(\omega)$ and effective distribution functions $f_{\alpha}\left(\omega ; l_{3}\right)$ for (a) a monatomic slab-shaped crystal and $(\mathrm{b})-(\mathrm{h})$ a layered structure. In this figure, $M$ represents the masses of the particles; in the monatomic crystal, all particles have a mass of 1 , and in the layered structure there are particles of mass 1 and mass $\frac{1}{5}$. 
thick crystals, and each surface acts as a perturbation on the surface modes associated with the other surface.

Table I shows clearly that interface effects are small compared to surface effects. Even for a mass ratio of $1 / 5$, the change in $\langle\omega\rangle_{f} /\left\langle\omega^{-1}\right\rangle_{f}$ for a surface film due to the presence of the interface is only a few percent of the change due to the presence of the surface. This outcome is reasonable in view of the results of Housley and Hess, ${ }^{13}$ which imply the following ${ }^{14}$ :

$$
\left\langle\omega^{-2}\right\rangle_{f}^{-1 / 2} \leq\left\langle\omega^{-1}\right\rangle_{f}^{-1} \leq\langle\omega\rangle_{f} \leq\left\langle\omega^{2}\right\rangle_{f}^{1 / 2} .
$$

Also, if the mass $M_{f}$ of the film is varied, but the force constants and the position of the film within the layered structure are not changed, then

$$
\begin{gathered}
\left\langle\omega^{-2}\right\rangle_{f}^{-1 / 2} \propto M_{f}^{-1 / 2}, \\
\left\langle\omega^{2}\right\rangle_{f}^{1 / 2} \propto M_{f}^{-1 / 2} .
\end{gathered}
$$

Equations (9)-(11) suggest that

$$
\begin{aligned}
\left\langle\omega^{-1}\right\rangle_{f}^{-1} & \approx \text { const } \times M_{f}^{-1 / 2}, \\
\langle\omega\rangle_{f} & \approx \text { const } \times M_{f}^{-1 / 2} .
\end{aligned}
$$

If $\left\langle\omega^{n}\right\rangle_{\text {bulk }}$ is the moment for an infinite crystal with mass $M_{f}$, it is, of course, exactly true that

$$
\left\langle\omega^{n}\right\rangle_{\mathrm{bulk}}^{1 / n} \propto M_{f}^{-1 / 2} .
$$

Equations (10)-(14) imply that the mass mismatch at an interface will have no effect at all on $\left\langle\omega^{2}\right\rangle_{f} /$ $\left\langle\omega^{2}\right\rangle_{\text {oulk }}$ and is expected to have only a small effect on the other two quantities in the Table I. As mentioned above, however, an interface does have some effect in raising the vibrational frequencies of the lighter particles and lowering those of the heavier particles, in the sense that both $\langle\omega\rangle_{f}$ and $\left\langle\omega^{-1}\right\rangle_{f}^{-1}$ are, respectively, increased or decreased.

The main implication of the present results is that changes in the phonon frequencies due to interface effects are only a few percent of the changes produced by surface effects. Consequently, the change in frequencies produced by a surface and an interface is only about half the change produced by two surfaces - more specifically, about $40-50 \%$ for three-layer films with mass ratios ranging from $1 / 5$ to $5 / 1$.

This conclusion, of course, has been shown to hold only for a model in which the interface involves nothing more than a mass mismatch. In real films the situation will be complicated by mismatches in structure as well (not to mention differences in the effective ion-ion interaction and changes in the electronic properties). It is reasonable to expect, in fact, that the influence of structural mismatching between films is comparable in importance to that of disorder within the films, and that both types of disorder will have a substantial effect on the phonon frequencies in experimental films.

We wish to thank J. W. Garland, M. Strongin, and J. M. Dickey for useful conversations, and Dr. Garland for sending a preprint of his work. We are also indebted to Dr. D. P. Johnson for bringing the results of Ref. 13 to our attention.
*Work supported by the U. S. Air Force Office of Scientific Research under Grant No. AF-AFOSR 1257-67.

${ }^{1}$ M. Strongin, O. F. Kammerer, J. E. Crow, R. D。 Parks, D. H. Douglass, Jr., and M. A. Jensen, Phys. Rev. Letters 21, 1320 (1968).

${ }^{2}$ M. Strongin (private communication).

${ }^{3}$ W. L. McMillan, Phys. Rev. 167, 331 (1968).

${ }^{4} \mathrm{~J}$. W. Garland, K. H. Bennemann, and F. M. Mueller, Phys. Rev. Letters 21, 1315 (1968); J. W. Garland and $\mathrm{P} 。 \mathrm{~B}$ 。Allen (unpublished).

${ }^{5}$ T. T. Chen, J. D. Leslie, and H. J。T。 Smith, Phys . Rev. Letters 22, 526 (1969).

${ }^{6}$ J. M. Dickey and A. Paskin, Phys. Rev。 Letters 21 , 1441 (1968).

${ }^{7}$ R. E. Allen and F. W. de Wette, Phys. Rev. 187 , 883 (1969).

${ }^{8}$ For convenience we write " $\xi_{\alpha}\left(l_{3} ; \omega\right)$ " with the understanding that the $\xi_{\alpha}\left(l_{3} ; \omega\right)$ are distinguished by the identity of $\omega$ (i.e., the mode to which it corresponds) rather than the numerical value of $\omega$ (which can be the same for different modes).

${ }^{9}$ R. E. Allen and F. W. de Wette, Phys. Rev. 179 , 873 (1969).

${ }^{10} \mathrm{~A}$ finite number of sample frequencies (1701 independent frequencies) were used in the calculations of this paper, so there are some irregularities in the histograms of Fig. 2. The moments of Table I were calculated directly from the frequencies, rather than from these histograms. Being averages over all the frequencies, these moments are considerably more accurate than the histograms.

${ }^{11}$ This result is not shown here, but see R. E. Allen and F. W. de Wette, J. Chem. Phys. 51, 4820 (1969).

${ }^{12}$ One interesting question is the following: At an interface between particles of masses $m$ and $M$, in the limit $m \rightarrow 0$ will the interface have the same effect on the vibrations of the particles with mass $M$ as a free surface would have? The answer is "no." Equation (11) shows that the effective $\left\langle\omega^{2}\right\rangle$ for the particles with mass $M$ is independent of $m$; therefore this moment will always be larger at an interface than at a surface (see Table I), and so the frequency spectra at a surface and at an interface with massless particles are different.

${ }^{13}$ R. M. Housley and F. Hess, Phys. Rev. 146, 517 (1966).

${ }^{14}$ The moment $\left\langle\omega^{-2}\right\rangle_{f}^{-1 / 2}$ is actually zero in a free slab of infinite extent, but nonzero in the physically more realistic case where the periodicity lengths are finite in the $x$ and $y$ directions. See Ref. 9. 